\title{
Basic and translational research careers as early faculty cardiothoracic surgeons-perspectives from 2 young investigators
}

\author{
Keshava Rajagopal, MD, $\mathrm{PhD},{ }^{\mathrm{a}}$ and Bryan A. Whitson, $\mathrm{MD}, \mathrm{PhD}^{\mathrm{b}}$
}

\footnotetext{
From the ${ }^{\mathrm{a} C e n t e r}$ for Advanced Heart Failure and the Department of Cardiovascular/Thoracic Surgery, University of Texas-Houston and Memorial Hermann-Texas Medical Center, Houston, Tex; and ${ }^{\mathrm{b}}$ Division of Cardiac Surgery, Department of Surgery, The Ohio State University School of Medicine and Medical Center, Columbus, Ohio.

K.R. is the 2014 to 2016 recipient of the Norman E. Shumway Career Development Award of the International Society for Heart and Lung Transplantation.

Disclosures: Authors have nothing to disclose with regard to commercial support.

Received for publication Oct 21, 2015; revisions received Jan 22, 2016; accepted for publication Feb 24, 2016; available ahead of print April 13, 2016.

Address for reprints: Keshava Rajagopal, MD, PhD, Center for Advanced Heart Failure and Department of Cardiovascular/Thoracic Surgery, University of Texas-Houston and Memorial Hermann-Texas Medical Center, 6400 Fannin, Suite 2350, Houston, TX 77030 (E-mail: keshava.rajagopal@uth.tmc.edu).

J Thorac Cardiovasc Surg 2016;152:362-6

$0022-5223 / \$ 36.00$

Copyright (c) 2016 by The American Association for Thoracic Surgery

http://dx.doi.org/10.1016/j.jtcvs.2016.02.068
}

We provide advice for young faculty members who are interested in conducting basic and translational research within the context of clinically active careers in cardiothoracic surgery. This advice is based largely on our personal experiences-both successes and failures-but we have incorporated the observations and experiences of successful investigators as well. We have tried to organize our thoughts in the context of developing and completing a scientific project. This article does not include a section on actually conducting scientific studies, as this process differs for each project and each scientist (Figure 1).

\section{STEP I: PREREQUISITES FOR THE SURGEON- SCIENTIST}

All things are ready, if our minds be so.

-King Henry V, in Henry V (William Shakespeare)

Although our focus is on how new surgeons can initiate and develop research programs, the preparation needed to perform basic or translational research is worth discussing. Just as training is required to perform cardiothoracic surgical operations, it is required to perform basic and translational research. In our cases, we decided to pursue complete graduate-level scientific training prior to cardiothoracic surgery residency, and we obtained PhDs, which can be accomplished either before or during medical education, or during residency training.

One of us undertook graduate study in an $\mathrm{MD} / \mathrm{PhD}$ program and subsequently performed a postdoctoral research fellowship during general surgery residency; the other obtained a $\mathrm{PhD}$ during general surgery residency. In our opinion, this level of training was of great value. One's initial field of study does not have to be one's current area of study or expertise. However, if a recent shift in research

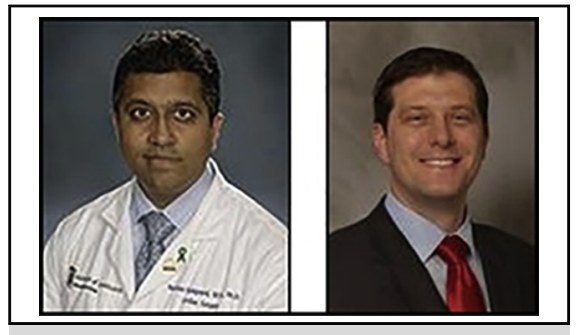

Keshava Rajagopal, MD, PhD, and Bryan A. Whitson, $\mathrm{MD}, \mathrm{PhD}$

\section{Central Message}

We provide advice for young faculty members who are interested in conducting basic and translational research within the context of clinically active careers in cardiothoracic surgery.

See Editorial Commentary page 367.

area has occurred, formal mentorship (see step III) may be required. Rather, the key elements of modern scientific training are generally applicable principles: (1) rational hypothesis formulation; (2) study/experiment conception or design; (3) study/experiment conduct; (4) analysis and interpretation of findings; (5) construction or modification of a mechanistic theoretical framework to explain the results of the study; and (6) construction of a scientific publication.

Obtaining a PhD—with constituent coursework and scientific research-certainly provides a valuable foundation for a scientific career, but it is not requisite. Specifically, formal didactic coursework is not necessary to perform scientific research of even the highest caliber. Yet, most successful scientists who are surgeons or clinicians as well have a track record of conducting research during their undergraduate education, medical/graduate school, and residency/fellowship training. The last of these phases is perhaps most important in the context of cardiothoracic surgery, because: (1) engaging in research that is closely related to one's clinical field is sensible and likely interesting (see later section on); and (2) historically, our training period has been among the longest of all medical specialties (ie, not conducting research during residency/fellowship usually means taking a long hiatus from scientific research). 

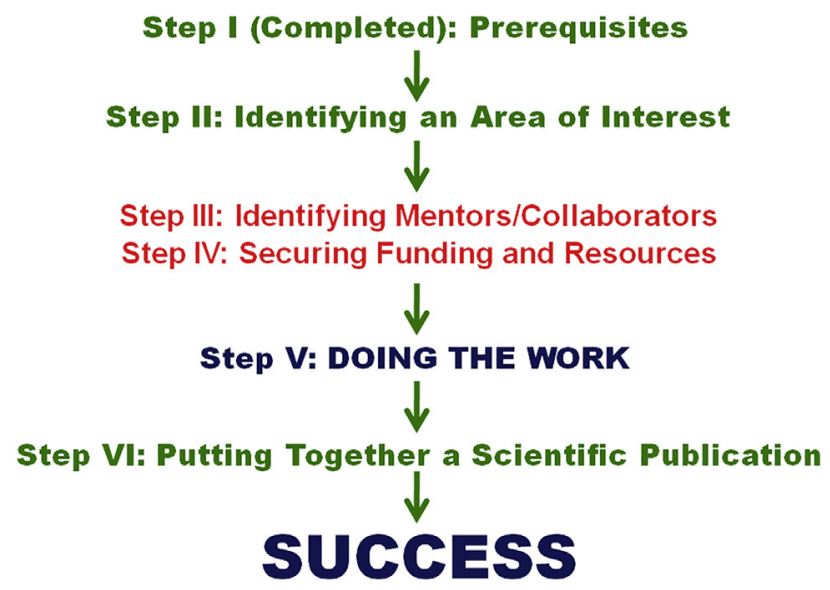

FIGURE 1. A pathway for early research success for the early investigator: Steps highlighted in green, in our opinion, are comparatively less difficult to accomplish, compared with steps highlighted in red.

However, the relatively new residency training paradigms may affect the development of cardiothoracic surgeon-scientists. On one hand, a shortened training period permits allocation of 2 or more years to research for many trainees. On the other hand, comparatively few (eg, Stanford University, the University of Pennsylvania, and the University of Maryland) residency programs have well established pathways for research to be conducted in the context of integrated training. At least some residents in integrated residency training programs may have chosen to enter such programs to "save time." The career trajectories of these trainees (ie, academic vs private practice) is not known, because few have graduated to date. Thus, the impact of integrated residency training programs on developing cardiothoracic surgeon-scientists is unclear.

\section{Key Point}

- Previous, particularly recent, scientific experience is essential for early career cardiothoracic surgeonscientists.

\section{STEP II: IDENTIFYING AN AREA OF INTEREST AND SCIENTIFIC PROJECTS}

\author{
They that apply themselves to trifling matters \\ commonly become incapable of great ones. \\ —Francois de la Rochefoucauld
}

This quote illustrates the key basis for identifying areas of interest, and within this, specific scientific questions to attempt to answer. Broad areas of interest relate to things that, for the most part, we cannot explain-ie, are matters of taste. Are you interested in heart failure? Or lung failure? Or vascular biology? Or cancer biology? The first question that the early investigator must ask is: "What do I find interesting?" or "What do I like?" We have placed this section before the section on mentors and collaborators, because before choosing others with whom to work, who already have expertise in specific areas, early investigators should identify what their interests are.

Scientific research relates to answering questions to which answers are unknown. The quote provides us with the notion that, as investigators, choosing important questions to address is essential. This opinion, however, is merely one of many-one can achieve "success" in terms of publications, career advancement, etc.-while studying problems that are neither scientifically deep nor of tremendous clinical relevance. But doing something that is deeply personally fulfilling, of broad interest to both you and the scientific community at large, and of societal value, means addressing scientifically deep and clinically important problems. For an individual problem to satisfy both conditions, in the context of an individual investigator performing both scientific research and clinical surgery, scientific and clinical interests need to be closely aligned.

A problem that is clinically important is relatively easy to identify, because all one has to ask is "With what do we struggle in our field?" Identifying a scientifically deep/ fundamental problem is somewhat more difficult, because it requires having a comprehensive understanding of the background and current state of the area of interest, to understand core knowledge gaps. A deep level of understanding is needed to formulate and answer scientific questions, whereas many investigators have a superficial understanding. Thus, young investigators should study a subject in which they have a solid knowledge base, which ties into the "prerequisites" already discussed. In summary, choosing an area that is linked to both your previous scientific background and current clinical practice is advisable. In this way, investigator enthusiasm, intellectual interest, and experiential foundation-and the chances of success-are maximized.

Although mentors and collaborators do provide valuable input (see later section on), the success or failure of a project depends greatly on your knowledge of the field. Early investigators need to be master strategists, not tacticians, or what is worse, foot-soldiers. A solid combination of motivation, and clinical and scientific background and interests, enhances the likelihood of success. If early investigators are critically dependent on others for idea generation, rather than execution of ideas, they: (1) may not develop scholarship or expertise in the field; (2) may not develop their interest because of being less intellectually engaged; and (3) will not become scientifically independent. We advise safeguarding against such pitfalls by choosing areas of study and specific problems in which you have genuine interest, and preferably a solid background.

However, although enthusiasm, intellect, and knowledge are necessary, they are not sufficient to achieve success. 
Although theoretical research (eg, mathematical modeling in physiology) is not limited by infrastructure and other resources, experimental research is. Choosing a project requires understanding your individual resources, the resources of your mentors and collaborators, and institutional infrastructure.

\section{Key Points}

- Areas of research interest are subjective, but they should be chosen such that they are well aligned with clinical interests and expertise.

- Within an area of interest, choosing scientifically profound and clinically deep problems is most intrinsically interesting, valuable, and likely to be funded (see Step IV).

\section{STEP III: IDENTIFYING MENTORS AND COLLABORATORS}

Talent wins games, but teamwork and intelligence wins [sic] championships. -Michael Jordan

We need to touch upon a related topic, before we discuss mentors and collaborators: the job itself. How to choose the right job could be a topic for an entire American Association for Thoracic Surgery Annual Meeting or issue of this journal. Ideally, protected time and seed money should be ensured. How much protected time should be allotted? As much as needed, depending on the project(s), should be taken, while maintaining time to develop a clinical practice. The $80 \%$ that our nonsurgical counterparts typically have is probably neither appropriate nor realistic, but $20 \%$ is almost certainly insufficient to be well invested in science. An approximately 50\% time commitment is reasonable, and is what the National Institutes of Health expects for K-level (eg, K08 or K23) grant recipients who are surgeons. But a number means little in isolation. Rather, having partners and mentors committed to your success, who understand the importance of your science to both you and your division and/or department, is more important. At times, doing an experiment or writing a grant or paper must take priority over doing a case. As to how much money should be allotted ... as much as you can negotiate! More on this topic later.

Assuming that you have chosen the "right" job, let us now discuss building your research program-the championship-winning team. Researchers starting out in their careers as faculty members need help. The ability to identify and ask for specific needed assistance is critical to a successful career. Two rules are inviolable, in our opinion: Senior mentors or even collaborators should be of the highest quality scientifically and/or clinically, depending on the type of support provided; and mentors and collaborators should have skills or experiences that complement rather than mirror those of newer investigators. Playing a football game with a quarterback and a receiver, or a basketball game with a point guard and a center, wins games; playing with 2 quarterbacks or 2 point guards generally does not.

\section{Arranged Marriages}

As in the history of marriage, an arranged pairing is a method for choosing a mentor or collaborator with a long track record. With this approach, a highly limited range of prospective "suitors" is offered by the senior investigator to the early investigator, who then selects from the choices (assuming more than 1 is available). The investigator and mentor should have compatible but not necessarily identical research interests. The early investigator is often given office and research space within a senior investigator's laboratory. Such an arrangement is not a slight to the investigator's abilities, but rather is typically done with the best interests of both investigators in mind. Such relationships are made because the senior investigator has an established track record of funding (and thus resources that can be shared), has had successful collaborations, and/or is in a leadership position.

The upsides of these arrangements for early scientists are that they get access to supplies, equipment, and potentially labor help, that they would be unable to garner otherwise. Early investigators with less-firm scientific foundations might benefit from the mentor's intellectual input as well. The degree of early success, ie, data and publications in the first few years - typically is proportional to the degree of alignment, ie, shared scientific or clinical interest. However, as time progresses, if the mentor has done an appropriate job, the investigator develops independence, which may be aided by a clear distinction between the expertise of the early versus senior investigators. If a mentor is a senior cardiothoracic surgeon, the likelihood of a complete overlap of interests - and failure of the young faculty member to establish independence-is higher. In contrast, when a mentor is a distinguished scientist but not a cardiothoracic surgeon, scientific interests hold the relationship together, while lack of shared clinical interests maintains sufficient distinction, particularly with respect to the motivations for and specific foci of research.

\section{Dating}

This approach often results from not identifying a focused area of investigation, or from insufficient guidance from leadership. Yet, it can yield success. Early investigators may have an idea of what they want and need, and may attempt to get one or more senior individuals to help by supplying resources and/or time. For this approach to work, the newer faculty members need a clear 
idea of both goals and tasks - from their perspective as well as that of the mentors and/or collaborators. Accordingly, young investigators must tailor their strategy such that it becomes mutually beneficial, in contrast to the arranged approach, which presupposes a priori alignment of interests. Often, early investigators and collaborators are introduced by a mutually interested third party, who can facilitate the relationship as well.

A "speed dating" approach to research relationships has been in vogue recently. Two or more departments that have decided or been mandated to form collaborative partnerships to strengthen relationships, or demonstrate institutional cohesiveness, are involved. In this approach, faculty members from a department physically meet, and faculty members from other departments rotate through. Each pair has a 5- to 8-minute conversation, with each of the participants trying to convey what they have to offer. At the end, interested parties follow up with an e-mail or phone call to arrange further discussions. The short- and long-term success rates of these events are unclear.

The key to success of the early-senior investigator relationship is one of mutual respect and mutual gain-ie, symbiotic "mutualism." Both parties must do their part, contribute, and have vested interests. Seldom does one individual have a single, completely altruistic mentor or only one key collaborator. As one's science evolves and questions expand, the need expands for multiple mentors and/or collaborators. What is paramount to the successes/ failures of these relationships is that the early investigators remember that the relationships need to be mutually beneficial.

\section{Key Points}

- Mentors and collaborators should be well regarded experts in the fields in which they are providing support to early investigators.

- Mentors and collaborators should have backgrounds that complement rather than mirror those of early investigators.

- For scientific relationships to succeed, in both the short and long term, they must be of mutual benefit to early investigators and mentors and collaborators.

\section{STEP IV: SECURING FUNDING AND RESOURCES}

Never, never, never give up.

$$
\text { —Winston Churchill }
$$

Securing monies and resources (personnel, space, or equipment) are ongoing struggles of basic and translational research endeavors; translational research in particular is more resource intensive than basic research. Unlike with steps I to III, early researchers have the least control in this arena, particularly with respect to extramural funding. Many approaches can be used to secure these necessities to perform research. The first is to adopt the "arranged marriage" approach, in which an early investigator can participate in a well-funded senior investigator's laboratory. When done well, this arrangement can be mutually beneficial. If successful, this approach allows an early investigator the opportunity to collect preliminary data and acquire a track record of publication.

Early independence, with "dating" of collaborators, is a reasonable approach, but it requires a more-extensive startup package for the early investigator and relies on previous recent work as the source of preliminary data for grant applications. Initial recruitment packages are varied. As discussed earlier, ideal early investigators have a track record of success, either from before their residency or from dedicated laboratory time during their residency. Such early investigators have more options regarding strategic approaches to beginning a research program.

An important aspect of discussions among early investigators, mentors and collaborators, and administrators is overall recognition of monetary and personnel expenses required, how they will be allocated, and for what duration. Early investigators need a modest understanding of the level of initial funds needed for the proposed science. They should ensure that money and personnel to be used for science, the duration of the project, and the source be delineated in writing at the onset. A common approach in many large institutions is to provide core facilities and space for early investigators to use. As much as possible, these stipulations should be identified ahead of time. For example: Will early investigators be able to use the facilities at cost? Do they have to pay "rent" for space? If a technician is shared, how much time is allotted, who pays for the time, and what are the expectations? Personnel are at least as important as cash funds.

Once initial conditions are set, all available funding sources must be considered. Unless a stellar startup package is in place, akin to that of a primary research faculty member in a nonsurgical department, the major funding source, even early in the process, is nondepartmental. Various options, in decreasing order of proximity to the home institution, and thus in increasing order of difficulty, are as follows: (1) institutional, nondepartmental funding mechanisms for young faculty members, ie, "starter" grants; (2) grants from a local chapter of a national society, or local/regional philanthropy; (3) grants or career development awards from a national society; and (4) grants from a federal research agency (eg, National Institutes of Health, Department of Defense, National Science Foundation) grants. With respect to the last of these, at large institutions, "center" grants may be awarded, from which annual funds are available to fund a selected number of young faculty members as grant awardees. These grants (eg, "institutional 
K") are equivalent to federal-level grants monetarily, but they are somewhat easier to obtain.

Regardless of surgical hubris and prior track record, early investigators are unproven, untested "commodities" in the real world of academic medicine, in which they must eventually function independently. They must demonstrate early success and continue to contribute to the success of the "team" and institution before individual success is likely to be realized. Never forget Churchill, and never, never, never give up! Success is around the corner.

\section{Key Points}

- Early investigators need to consider numerous funding options (as described in sections I to IV, above).

- Be persistent in applying for grants.

\section{STEP V: DOING THE WORK ...}

\section{STEP VI: PUTTING TOGETHER A SCIENTIFIC PUBLICATION}

There is no value in anything until it is finished. -Genghis Khan

Once a project (or "story" within a project) is completed, its culmination is in communicating the findings to the scientific public. We summarize 2 useful approaches to constructing scientific manuscripts.

\section{Constructing a Paper From Beginning to End}

This approach works well for the more experienced scientist-particularly one with grant-writing experience. As with other forms of writing, scientific papers can be used to tell stories. The "beginning to end" approach proceeds linearly, starting with background information (ie, existing theory). The problem and central hypothesis are framed next, in terms of missing information and/or unresolved questions within the existing framework. Next, the experimental methodology is summarized, followed by presentation of the scientific findings. Results can be organized in various ways.

One approach centers around methodology - the initial results presented relate to demonstration of the validity of one or more experimental approaches employed, followed by the results of experiments that test the central hypothesis and/or address questions of interest. Another approach organizes the results on a spectrum from fundamental to applied, ie, from the most basic scientific findings to those with the greatest clinical relevance; this builds the story upon a foundation of basic principles. Finally, another common approach presents the results in chronological order- "first, we did this set of experiments, then because of what we found, we proceeded to the next set of experiments...." However, this approach is appropriate only when the studies are conducted in a logically sequential fashion; in real life, such an ordering may not have occurred. The paper ends with a discussion section, in which the findings are briefly summarized, and conclusions are drawn. Either the conclusions are fitted into the existing conceptual/theoretical framework, or the framework is modified based on the results. Questions are raised in the context of the conclusions drawn from the results.

\section{Constructing a Paper "Based on the Figures"}

This approach is easier to implement for the less experienced scientist. Figures that comprehensively present the experimental findings are constructed. Then, the results section is constructed from the figures, using one of the strategies outlined earlier. The methods section is added. The major difference between this approach and the "beginning to end" approach is that the introduction is generated subsequent to the results section, even though it is presented first. That is, the problem is framed retrospectively. This strategy is useful for the novice scientist, to whom the existing conceptual framework and knowledge gap into which the studies fit might not be readily apparent.

Another advantage of the "based on the figures" approach is that it rapidly identifies additional experiments that are needed to complete a scientific story, before the writer commits time to the remainder of the paper. Finally, the discussion section is constructed. Although the discussion can be constructed as outlined earlier, because it is generated immediately after the introduction, discussion sections created using the "based on the figures" approach tend to be less speculative and more limited in scope-either explaining the data in the context of existing theory, or modifying or rejecting the existing theoretical framework as a result of the studies performed.

\section{Key Point}

- Scientific publications are the tangible end-products of research endeavors, and they can be constructed using 1 of at least 2 standard approaches.

\section{CONCLUSIONS}

Early cardiothoracic surgeons interested in pursuing basic and translational research face several challenges. Building a clinical practice and foci of expertise, while doing the same in highly time- and resource-intensive areas of research, requires firm scientific and clinical foundations, motivation, time, resources, and luck. We have chosen this path for ourselves, and would do so again, without hesitation. Our hope is that the thoughts we have outlined will aid our colleagues in these pursuits. 